\title{
Research Paper: Comparing Psychometric Properties of GLFS-5 With GLFS-25 for Screening Locomotive Syndrome in Community-dwelling Iranian Older People
}

\author{
Nasim Sadeghi Mahalli' ${ }^{1}$, Mohammad-Ali Hoseini² (D), Mehdi Rahgozar ${ }^{3}$ (D) Kian Norouzi Tabrizi $^{4^{*}}$ (D) \\ 1. Department of Nursing, University of Social Welfare and Rehabilitation Sciences, Tehran, Iran. \\ 2. Department of Rehabilitation, University of Social Welfare and Rehabilitation Sciences, Tehran, Iran. \\ 3. Department of Biomedical and Computer Sciences, University of Social Welfare and Rehabilitation Sciences, Tehran, Iran. \\ 4. Department of Nursing, Iranian Research Center on Aging, University of Social Welfare and Rehabilitation Sciences, Tehran, Iran.
}

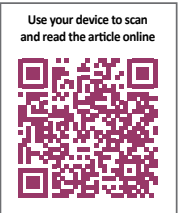

Citation Sadeghi Mahalli N, Hoseini MA, Rahgozar M, Norouzi Tabrizi K. Comparing Psychometric Properties of GLFS5 With GLFS-25 for Screening Locomotive Syndrome in Community-dwelling Iranian Older People. Iranian Rehabilitation Journal. 2021; 19(4):417-424. http://dx.doi.org/10.32598/irj.19.4.875.2

http://dx.doi.org/10.32598/irj.19.4.875.2

Article info:

Received: 09 Nov 2020

Accepted: 20 Sep 2021

Available Online: 01 Dec 202

Keywords:

Locomotion disorders, Screening tools, Elderly

\section{A BSTRACT}

Objectives: Locomotive syndrome refers to reduced mobility due to impairment of locomotive organs. Because of the importance of screening locomotive syndrome among older people, this article is focused on psychometric characteristics of Geriatric Locomotive Function Scale (GLFS)-5 and comparing it with GLFS-25 in Iranian older adults

Methods: This research was conducted on 320 older Iranian people. Validity and reliability of the GLFS-5 were examined and confirmed using Content Validity Index (CVI), factor analysis, correlation coefficient with the European Quality of Life Scale-5 Dimension (EQ5D) questionnaire, the Cronbach $\alpha$ value for internal consistency, and intraclass correlation and Receiver Operating Characteristic (ROC) technique to determine the cutoff score for the locomotive syndrome.

Results: In phase 1 of the study, 250 Iranian older people $\geq 60$ years were analyzed. The factor analysis showed that the GLFS-25 is a multi-dimensional scale (Activities of Daily Livings [ADLs] and Quality of Life [QoL], pain, social relationship, and psychological status), and GLFS-5 is a one-dimensional scale (the ability to perform daily activities). The Cronbach $\alpha$ values for GLFS-25 and GLFS-5 were 0.93 and 0.84, respectively. As for association between the GLFS-25 and GLFS-5 with EQ-5D and Visual Analogue Scale (VAS) (health selfperceived), the Pearson correlation coefficients were 0.85 and -0.72 for GLFS-25 ( $\mathrm{P}=0.01)$ and 0.82 and -0.67 for GLFS-5 ( $\mathrm{P}=0.01$ ), respectively. The cutoff scores to identify locomotive syndrome for the GLFS-25 and GLFS-5 were 16 and 4, respectively.

Discussion: Considering the good validity and reliability properties of the GLFS-5 compared to GLFS-25 and the more convenient use of this short version of the GLFS, its application is highly recommended for community-based screening of locomotive syndrome in Iranian older people.

\section{* Corresponding Author:}

Kian Norouzi Tabrizi, PhD.

Address: Department of Nursing, Iranian Research Center on Aging, University of Social Welfare and Rehabilitation Sciences, Tehran, Iran. Tel: +98 (912) 2073826

E-mail:drkian_nourozi@yahoo.com 


\section{Highlights}

- Early and detective screening of locomotive syndrome requires reliable, standard, and user-friendly tools.

- The psychometric properties of both versions of the GLFS tool (25 and 5 items) are very close and acceptable.

- So, the short version (GLFS-5) can be used instead of the long version in emergencies to screen seniors.

\section{Plain Language Summary}

The locomotive syndrome is one of the most common disorders that can lead to dependency and limited activity daily living among seniors. Early detection of this condition requires screening tools that fit seniors. The questionnaire of Geriatric Locomotive Function Scale (GLFS) is one of the instruments to identify the elderly persons at locomotive syndrome risk. It has been designed in two versions ( 5 and 25 items) by the Japan Orthopedic Association. To ensure the applicability and useability of this questionnaire in the Iranian older people, a psychometric review is necessary. Because the time saving and accuracy of screening in older people is very important, and no studies have compared psychometric properties of two versions of the GLFS, this research was done on a representative sample of community-dwelling Iranian older people. Comparison of psychometric properties of the two versions shows that both have good validity and reliability among Iranian seniors. Also, due to the proximity of the psychometric properties, we recommend using the short version in emergencies to save time.

\section{Introduction}

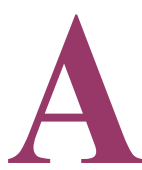

ging, as a worldwide phenomenon, is the result of reduced mortality in human communities, primarily due to more healthy lifestyles and more access to quality health care systems [1]. During this transition, chronic conditions such as musculoskeletal disorders have been introduced as main health concerns, especially in older adults [2].

The Japanese Orthopedic Association (JOA) has proposed the concept of the locomotive syndrome (LS), which refers to 1) conditions that will require due care and consideration, or 2) high-risk conditions that need nursing long care soon because of locomotive organ dysfunction in the older people [3]. The prevalence of LS among seniors has been reported as $16 \%$ to $40 \%$ in various studies $[4,5]$. According to some studies, $44 \%$ to $60 \%$ of the Iranian older people have physical and locomotive disabilities $[6,7]$.

LS is one of the most critical issues that can reduce the quality of life and disable older people [8]. Besides, there is a relationship between LS and mental disorders among older people [9]. Studies in Iran show that one of the main reasons to visit the medical centers and clinics among seniors is physical disability and locomotive disorders $[10,11]$.
One of the most important issues in this context is evaluating the locomotive function of at-risk persons with appropriate screening scales. One of these instruments is the Geriatric Locomotive Function Scale (GLFS) developed by the Japanese Orthopedic Association in 2012. The GLFS was published in two versions: the GLFS-25 and GLFS-5, in Japanese and English [12]. The GLFS-25 includes 4 questions about pain, 16 questions about Activities of Daily Living (ADL) and Quality of Life (QoL), 3 questions related to social activities, and 2 questions related to the mental condition of older people [13]. The GLFS-5 is a brief version of the GLFS-25. This version was developed by Akaike Information Criteria (AIC) and named the quick-version. Because it is essential to use easy, accurate, and quick screening among older people, a short version, GLFS-5, can be helpful [14]. So, the present study was conducted to compare the psychometric properties of GLFS-5 with the GLFS-25 tool for screening LS in community-dwelling Iranian older people.

\section{Materials and Methods}

The proposal of our methodological study has been approved by the IRB of the University of Social Welfare and Rehabilitation Sciences, Tehran, Iran. After explaining the research goals, informed consent was obtained from all participants. The study data were collected from the seniors in various urban places such as gardens, market places, mass gatherings, and so on (from three cities of Tehran, Qaemshahr, and Sari). The samples were pur- 
posively selected based on inclusion and exclusion criteria from July 2017 to August 2017. The GLFS in the full version is a self-administered questionnaire consisting of 25 items (4 questions for pain, 16 questions for activities of daily living, 3 questions related to social functioning, and 2 questions related to mental health status during the last month). These 25 items are rated with a 5-point Likert scaling, from no impairment ( 0 points) to severe impairment (4 points). The total score is between 0 and 100. The higher score is associated with worse locomotive function. The GLFS-5 includes questions of 12, 13, 15, 17, and 19 from the GLFS-25 questionnaire (Table 1). The international quality of life assessment model (Keller, 1998) was used for the translation and cultural adaptation of GLFS-25.

For evaluating the face validity of the questionnaire, 10 elders from the samples scored the necessity and importance of each item. For content validity, 10 experts (Geriatricians, Gerontology Nursing, and Physical therapists) scored the relevance of the items with the core concept of the questionnaire, and its Content Validity Index (CVI) was calculated for the questionnaire. Construct validity using confirmatory factor analysis, concurrent criterion validity using the Pearson correlation coefficient, and reliability of the tool were assessed based on internal consistency (Cronbach $\alpha$ coefficient) and test-retest (intraclass correlation). In the second phase of the study, the GLFS-5 cutoff score was established in Iranian community-dwelling seniors.

In this study, the inclusion criteria were men and women aged 60 years and older, able to read and write Farsi (official language in Iran) and answer questions without help (checked with abbreviated mental test). Also, they signed an informed consent form to participate in our study. We excluded the seniors who cannot walk without assistance, seniors with severe neurologic, cardiovascular, pulmonary, or renal disease, or with a history of mental illness and traumatic or pathologic fractures of the lower extremities and or spine within the preceding 6 months [14].

For all participants, data related to the following aspects were collected: history of chronic diseases, chronic pain (for 6 months and more), number of falls in the past year, European quality of Life Questionnaire (EQ-5D), numeric self-rated health status [Visual Analogue Scale (VAS)], and the GLFS-Farsi version. Content validity was measured by CVI. In factor analysis, 10 participants were recruited for every item in the tools (250 elders for the GLFS-25 and 50 elders for the GLFS-5). Concurrent validities of the GLFS-5 and GLFS-25 were assessed with EQ-5D and self-rated status instruments (VAS) and calculation of the
Pearson correlation coefficient; 120 elders participated in this phase. We used relevant GLFS-25 and GLFS-5 scores of 30 participants for calculating the Cronbach $\alpha$ coefficients and scores of 35 participants in a two-week interval for the intraclass correlation coefficient.

After confirming the validity and reliability of two Persian versions of scales and, in the second phase of the study, we investigated the cutoff point of the questionnaires. At first, the seniors were evaluated by primary physicians in community health centers (reviewing their health records in SIB Medical Records, i.e., the integrated health system public health database of the Ministry of Health, and conducting physical examinations). Then, they filled the screening questionnaires (GLFS-25/ GLFS-5). The cutoff scores were established by examining corresponding sensitivities and specificities and receiver operating characteristic (ROC) curve. After being evaluated by tests and clinical examinations, including two-step test, TUG (timed up and go), one-leg standing, Berg balance scale, and electronic medical records through the Ministry of Health System (SIB system), the qualified people who visited the medical clinic filled the GLFS-25 and GLFS-5 questionnaires.

SPSS v. 23 was used for all statistical analyses, including descriptive and analytical statistics (correlation coefficients, the Cronbach $\alpha$, and ROC curve). The process of examination and confirmation of validity and reliability of the GLFS-25 has been done by the researcher in the previous research [15]. In this article, we compared the psychometric properties of the GLFS-5 and GLFS-25.

\section{Results}

The study was done in two separate phases. A total of 320 community-dwelling seniors participated in this study (Table 1). In the first phase, data were obtained for analysis from 250 participants (168 men, 82 women; Mean \pm SD age: $69.68 \pm 7.8$ years, ranged: 60 -91 years). The CVI values of the GLFS-25 and GLFS5 were 0.856 and 0.88 , respectively. The factor analysis showed that the GLFS-25 is a multi-dimensional scale and consists of four factors (daily activities and quality of life, pain, social relationships, and psychological status), and the GLFS-5 is a one-dimension scale and consists of only one factor (the ability to perform daily activities). Based on the examination of the total variance, four factors in GLFS-25 explained $68.5 \%$ of the total variance of data, and the sole factor in GLFS-5 has explained $58.6 \%$ of the variance. The Pearson correlation coefficients of the GLFS-25 with the EQ-5D questionnaire and VAS scale were estimated as +0.86 and -0.72 , respectively 
Table 1. Characteristics of the participants in phase 1 and 2

\begin{tabular}{|c|c|c|}
\hline \multicolumn{2}{|c|}{ Variables } & \multirow{2}{*}{$\begin{array}{c}\text { No. (\%) } \\
108(23.8)\end{array}$} \\
\hline Su & Female & \\
\hline Jex & Male & $212(66.3)$ \\
\hline \multirow{7}{*}{ Age groups (y) } & $60-64$ & $91(28.4)$ \\
\hline & $65-69$ & $85(26.6)$ \\
\hline & $70-74$ & $51(15.9)$ \\
\hline & $75-79$ & $50(15.6)$ \\
\hline & $80-84$ & $25(7.8)$ \\
\hline & $85-89$ & $16(5)$ \\
\hline & 90 to up & $2(0.6)$ \\
\hline \multirow{3}{*}{ Marital status } & Single & $112(3.8)$ \\
\hline & Married & $242(75.6)$ \\
\hline & Other & $66(20.6)$ \\
\hline \multirow{3}{*}{ Education } & Diploma of high school & $274(85.6)$ \\
\hline & Associate degree & 20(6.3) \\
\hline & Bachelor's degree and upper & $26(8.1)$ \\
\hline \multirow{6}{*}{ Work status } & Government's employee & $13(5.2)$ \\
\hline & Business working & $44(17.6)$ \\
\hline & Housekeeper & $50(20)$ \\
\hline & Retired & $126(50.4)$ \\
\hline & Worker & $4(1.6)$ \\
\hline & Farmer & $13(5.2)$ \\
\hline
\end{tabular}

Iranian Rehabilitation Journal

$(\mathrm{P}=0.01)$. These figures for GLFS-5 questionnaires were +0.83 and -0.67 , respectively $(\mathrm{P}=0.01)$. The internal consistency (the Cronbach $\alpha$ ) for the GLFS-25 and GLFS-5 were calculated as 0.93 and 0.84 , respectively. Stability of scores as an indicator of reliability examined and confirmed for two scales as follows: The Pearson' correlation coefficient between test-retest (Interval time $=2$ weeks) for the GLFS-25 (Normal parameters: Shapirowilk Test: $\mathrm{W}_{(35)}=0.947, \mathrm{P}=0.094$ ) and GLFS-5 (Normal parameters: Shapiro-wilk Test: $\left.\mathrm{W}_{(35)}=0.943, \mathrm{P}=0.063\right)$, were 0.96 and 0.89 respectively, $(\mathrm{P}<0.01)$. Table 2 presents the reproducibility of all items.

Phase two of the study aimed to examine and establish cutoff scores for the GLFS-25 and GLFS-5 in Iranian elders. we recruited 70 elders for this phase of our study (Mean \pm SD age: $70.44 \pm 7.35$; female: 26 , male: 44 ). At this stage, the gold standard was the final diagnosis by a specialist physician. Based on our available data, the cutoff point for screening LS was established for two scales as follows: 16 for the GLFS- 25 (sensitivity $=0.88$, specificity $=0.84$ ) and 4 for the
GLFS-5 (sensitivity $=0.81$, specificity $=0.80$ ) among the Iranian community-dwelling older people (Tables 3 and 4).

\section{Discussion}

In this study, we calculated and compared the psychometric properties of the two questionnaires of GLFS-25 and GLFS-5 among Iranian older people. The psychometric indexes of the GLFS-5 showed that this brief version has good reliability and validity for screening LS in Iranian older people and is comparable with GLFS-25. The results of this study are similar to the original research in Japan [14].

According to the results of our previous study, GLFS25 has high reliability among Iranian older people [15]. The current study also showed that the reliability of the GLFS-5 questionnaire was good and comparable with GLFS-25. The factors affecting the reliability of scales are as follows: the quality of items in the questionnaire, the sequencing of the items, the number of questions, the 
Table 2. Reproducibility of each item for GLFS-25 and GLFS-5

\begin{tabular}{|c|c|c|c|c|}
\hline \multicolumn{3}{|c|}{ GLFS-25 } & \multicolumn{2}{|c|}{ GLFS-5 } \\
\hline Items & $\begin{array}{c}\text { Test-Retest Interclass } \\
\text { Correlation }\end{array}$ & $95 \% \mathrm{Cl}$ & $\begin{array}{c}\text { Test-Retest Interclass } \\
\text { Correlation }\end{array}$ & $95 \% \mathrm{Cl}$ \\
\hline 1 & $0.957^{* *}$ & $0.902-1.000$ & & \\
\hline 2 & $0.961^{* *}$ & $0.910-1.000$ & & \\
\hline 3 & $0.870 * *$ & $0.790-0.930$ & & \\
\hline 4 & $0.763^{* *}$ & $0.631-0.856$ & & \\
\hline 5 & $0.825^{* *}$ & $0.695-0.921$ & & \\
\hline 6 & $0.795^{* *}$ & $0.649-0.887$ & & \\
\hline 7 & $0.947^{* *}$ & $0.852-1.000$ & & \\
\hline 8 & $0.929 * *$ & $0.843-0.980$ & & \\
\hline 9 & $0.736^{* *}$ & $0.588-0.836$ & & \\
\hline 10 & $0.671^{* *}$ & $0.476-0.834$ & & \\
\hline 11 & $0.687^{* *}$ & $0.408-0.868$ & & \\
\hline 12 & $0.921^{* *}$ & $0.863-0.965$ & $0.493^{* *}$ & $0.157-0.955$ \\
\hline 13 & $0.868 * *$ & $0.766-0.932$ & $0.871 * *$ & $0.769-0.936$ \\
\hline 14 & $0.596 * *$ & $0.219-0.860$ & & \\
\hline 15 & $0.944 * *$ & $0.859-0.984$ & $0.944 * *$ & $0.875-0.986$ \\
\hline 16 & $0.949 * *$ & $0.873-1.000$ & & \\
\hline 17 & $0.928 * *$ & $0.866-0.974$ & $0.928 * *$ & $0.856-0.972$ \\
\hline 18 & $0.732^{* *}$ & $0.353-0.906$ & & \\
\hline 19 & $0.851^{* *}$ & $0.667-0.947$ & 0.851 & $0.643-0.945$ \\
\hline 20 & $0.898^{* *}$ & $0.767-0.976$ & & \\
\hline 21 & $0.891^{* *}$ & $0.839-0.950$ & & \\
\hline 22 & $0.868^{* *}$ & $0.558-0.978$ & & \\
\hline 23 & $0.921^{* *}$ & $0.776-0.979$ & & \\
\hline 24 & $0.862^{* *}$ & $0.677-0.967$ & & \\
\hline 25 & $0.640^{* *}$ & $0.306-0.856$ & & \\
\hline
\end{tabular}

** Correlation is significant at the 0.01 level (2-tailed).

Iranian Rehabilitation Journa

variation in the demographics of the participants, and the mindset and psychological status of the respondents [16]. It seems that the main contributing factor to the lower reliability for the GLFS-5 in our study is merely related to its brevity and fewer questions.

We used factor analysis to examine and verify the dimensionality of our Iranian versions of GLFS-25 and
GLFS-5. Factor analysis is a mathematical method that explores the internal correlations of the items in a questionnaire and extracts corresponding factors. Based on our factor analysis, the GLFS-25 consists of the following four factors: pain (items 1-4), ADL/QOL (items 5-21), social relationships (items 22,23), and mental health status (items 24,25). The GLFS-5, as a brief version of GLFS-25, has been developed using the Akaike 
Table 3. GLFS-25 cutoff score

\begin{tabular}{ccccc}
\hline Scores & Sensitivity & Specificity & $\mathbf{1}$ - Specificity & Youden Index \\
\hline 14 & 1 & 0.577 & 0.423 & 0.577 \\
15 & 0.977 & 0.692 & 0.308 & 0.669 \\
16 & 0.886 & 0.846 & 0.154 & 0.732 \\
17 & 0.773 & 0.885 & 0.115 & 0.658 \\
18 & 0.705 & 0.885 & 0.115 & 0.590 \\
19 & 0.659 & 0.923 & 0.077 & 0.582 \\
\hline
\end{tabular}

Information Criterion (AIC) method by the Japan research team [14]. Based on our results, it was revealed that the GLFS-5 questionnaire mainly consisted of the ADL factor of GLFS-25.

Concurrent validity evidence was provided by a high and statistically significant correlation between GLFS25/GLFS-5 and EQ-5D scores (0.86 and 0.83). In this study, the GLFS-25 cutoff score for detecting LS in Iranian elders appointed as scores $\geq 16$, which is the score determined for Japanese counterparts. On the other hand, we yield a different GLFS-5 cutoff score for older Iranian people than one in the Japanese study (4 vs 6). A nationwide study done by the Ministry of Health in Iran has shown that $24 \%$ of seniors have musculoskeletal problems and $71 \%$ chronic diseases and disabilities [17]. We didn't find any study about this issue.

Noge et al (2017) evaluated Locomotive Syndrome in older Japanese females with loco-check questionnaire [18]. Loco-check, a 7-item questionnaire, is useful for estimating the severity of the locomotive syndrome. The results showed that higher scores on loco-check were significantly correlated with smaller thigh muscle mass (a major measure of physical performance), poor nutritional status, and quality of life. This score was also significantly larger in the participants experiencing fallings, fractures, and lumbar pain than those without these adverse episodes [18].

Ishibashi (2018), states that with acceleration of population aging in Japan; prevention and treatment of diseases in the locomotor system and maintenance of motor function are important measures for extending healthy life years and gearing down the escalating demand for long-term care and rehabilitation services [19]. Adopting an active lifestyle, balanced and healthy nutrition, and screening for locomotion-related diseases are efficient preventive measures for LS. The JOA, therefore, proposed a set of exercises for LS called locomotion training comprising standing on one leg with the eyes open, squats, heel raises, and front lunges to improve locomotor function and countermeasure locomotive syndrome [19].

Fujita et al. (2018), investigated the association between two pathologies, locomotive syndrome and Lumbar Spinal canal Stenosis (LSS) [20]. A total of 200 participants comprising 120 men and 80 women, were enrolled in this

Table 4. GLFS-5 cutoff score

\begin{tabular}{ccccc}
\hline Scores & Sensitivity & Specificity & $\mathbf{1}$ - Specificity & Youden Index \\
\hline 2 & 0.955 & 0.500 & 0.500 & 0.455 \\
3 & 0.932 & 0.615 & 0.385 & 0.547 \\
4 & 0.818 & 0.808 & 0.192 & 0.626 \\
5 & 0.727 & 0.885 & 0.115 & 0.612 \\
6 & 0.545 & 0.962 & 0.038 & 0.507 \\
7 & 0.386 & 0.962 & 0.038 & 0.348 \\
\hline
\end{tabular}


study. Association of the severity of LSS (evaluated by Zurich claudication quesionnaire scores) was assessed with three LS risk tests (stand-up test, the two-step test, and a 25-question risk assessment) and TUG test. Logistic regression analysis revealed that LSS severity was positively correlated with the risk level of LS evaluated by the two-step test (OR=3.45, CI: 1.33-8.96) [20].

Endo (2016) investigated Osteoporosis, as a cause of locomotive syndrome [21]. Osteoporosis influences functional mobility and activities of daily living in older people. Patients with vertebral fractures and or first-time hip fracture are at high risk of a subsequent hip fracture [21].

Matsumoto et al (2016), reviewed screening, prevalence, causal and related factors, and the relationship between locomotive syndrome and falls and fractures in older adults with this syndrome [4]. They showed that the prevalence of the LS is significantly higher in women than in men, and this risk tends to increase markedly with advancing ages ( $\geq 70$ years). A more severe LS is related to knee pain, osteoporosis, sarcopenia, and lumbar disease. The incidence of falling in persons diagnosed with LS is higher than that of the older population in general [4].

Momoki et al (2016), investigated the relationship between sarcopenia, household status and locomotive syndrome among elderly women in Japan [22]. A total of 186 women aged over 65 years attending preventive care classes were enrolled in the study. Sarcopenia was identified in $21 \%$ of participants. Participants with sarcopenia were older, had a lower body mass index and calf circumference, were more likely to have LS, and living with their children and or grandchildren. In multivariate analysis, age, body mass index $<18.5 \mathrm{~kg} / \mathrm{m}^{2}$, and LS were significantly associated with sarcopenia, living with children and or grandchildren (OR 2.46, 95\% CI: 0.71-8.54), and dietary variety score $\geq 9$ (OR 4.98, 95\% CI: 0.97-25.56). Sarcopenia was associated with age, body mass index, dietary variety score, LS, and household status [22].

Kim et al (2016), conducted an outpatient cohort study on diagnostic abilities of two screening tools, the Lococheck and the GLFS-25 [23]. They investigated the associations of LS with clinical information, including the general status, orthopedic diseases, past medical histories, and exercise activities using a questionnaire survey. These analyses provide critical information to help clinicians decide whether to use the loco-check or GLFS-25 in various clinical conditions [23].

A healthy locomotive system is essential. Screening is a way to detect a locomotive syndrome in elders to have a disability-free life course. Screening and on-time diagnosis and management of locomotion diseases can preclude the advancement of functional disabilities. Many studies have shown that early diagnosis and management of LS has many desirable patient outcomes, such as alleviating chronic pain and improving physical functioning, social participation, and health-related quality of life.

\section{Conclusion}

GLFS is a standard method for measuring the health of the LS and monitoring the effectiveness of relevant interventions. The psychometric properties of both versions of the GLFS ( 25 and 5 items) show that both instruments have the proper validity and reliability characteristics in Iranian older people. The psychometric results of the short version (GLFS-5) show that its psychometric characteristics are very comparable to the long version (GLFS-25). Based on these findings and because using brief questionnaires is more convenient and feasible in elderly persons, we recommend the short version (GLFS-5) for older people with locomotive syndrome risk. Although the study provided valuable information about the validity and credibility of GLFS-5, it has some limitations. These limitations include time constraints on conducting and completing research and difficult access to representative samples. Therefore, further studies are recommended in larger populations and different communities.

\section{Ethical Considerations}

\section{Compliance with ethical guidelines}

This research was approved by the Ethics Committee of Tehran University of Social Welfare and Rehabilitation (Code: IR.USWR.REC.2017.367).

\section{Funding}

The project was sponsored by the Iranian Research Center on Aging University of Welfare and Rehabilitation Science.

\section{Authors' contributions}

All authors equally contributed to preparing this article.

\section{Conflict of interest}

The authors declared no conflict of interest. 


\section{References}

[1] United Nations. World population ageing 2009. New York: Department of Economic and Social Affairs; 2009. https:// www.un.org/en/desa/world-population-ageing-2009

[2] Gheno R, Cepparo J, Rosca C, Cotten A. Musculoskeletal disorders in the elderly. Journal of Clinical Imaging Science. 2012; 2:39. [DOI:10.4103/2156-7514.99151] [PMID] [PMCID]

[3] Akai M, Doi T, Seichi A, Okuma Y, Ogata T, Iwaya T. Locomotive syndrome: Operational definition based on a questionnaire, and exercise interventions on mobility dysfunction in elderly people. Clinical Reviews in Bone and Mineral Metabolism. 2016; 14:119-30. [DOI:10.1007/s12018-016-9210-8] [PMID] [PMCID]

[4] Matsumoto H, Hagino H, Wada T, Kobayashi E. Locomotive syndrome presents a risk for falls and fractures in the elderly Japanese population. Osteoporosis and Sarcopenia. 2016 2(3):156-63. [DOI:10.1016/j.afos.2016.06.001] [PMID] [PMCID]

[5] Silva T, Figueiredo M, Costa A, Rocha E, Borges L, Darder J. [Prevalence and factors associated with locomotive syndrome in community-dwelling older adults (Portuguese)]. Texto contexto-enferm. 2021; 30(e20200494):1-13. [DOI:10.1590/1980265X-TCE-2020-0494]

[6] Borhaninejad V, Momenabadi V, Hossseini S, Mansori T, Sadeghi A, Toroski M. [Health physical and mental status in the elderly of Kerman (Persian)]. Journal of North Khorasan University. 2014; 6(4):715-25. [DOI:10.29252/jnkums.6.4.715]

[7] Beiranvand R, Shokoohi S, Babanejad M, Behzadifar M, Delpisheh A. [Assessment of health status in elderly of Ilam province (Persian)]. Scientific Journal of Ilam University of Medical Sciences. 2014; 21(6):276-86. http:/ / sjimu.medilam. ac.ir/article-1-758-fa.html

[8] Hsieh CT, Yamazaki H, Wang J, Kamitani T, Yamamoto Y, Fukuhara S. Quality of life and disability-free survival in the elderly: The locomotive syndrome and health outcome in Aizu Cohort study. Journal of Aging and Health. 2021; 33(34):197-204. [DOI:10.1177/0898264320970323] [PMID]

[9] Nakamura M, Hashizume H, Nomura S, Kono R, Utsunomiya $\mathrm{H}$. The relationship between locomotive syndrome and depression in community-dwelling elderly people. Current Gerontology and Geriatrics Research. 2017; 2017:4104802. [DOI:10.1155/2017/4104802] [PMID] [PMCID]

[10] Nodehi Moghadam A, Ehsani Fard F. [Prevalence of physical impairment among the elderlly persons of the Tehran city in 1385 (Persian)]. Salmand: Iranian Journal of Ageing. 2007; 1(2):125-31. http:// salmandj.uswr.ac.ir/article-1-23-en.html

[11] Adib-Hajbaghery M, Akbari H. [The severity of old age disability and its related factors (Persian)]. Feyz. 2009; 13(3):225-34. https://www.sid.ir/en/journal/ViewPaper. aspx?ID $=164666$

[12] Tavares DRB, Santos FC. Locomotive syndrome in the elderly: Translation, cultural adaptation, and Brazilian validation of the tool 25-Question Geriatric Locomotive Function Scale. Revista Brasileira de Reumatologia. 2017; 57(1):56-63 [DOI:10.1016/j.rbre.2016.07.015] [PMID]

[13] Ning Z, Rui-li Z, Hui-juan L. [Validity and reliability of the chinese version of geriatric locomotive function scale (Chinese)]. Chinese Journal of Nursing. 2016. https://en.cnki. com.cn/Article_en/CJFDTotal-ZHHL201606032.htm
[14] Seichi A, Hoshino Y, Doi T, Akai M, Tobimatsu Y, Iwaya T. Development of a screening tool for risk of locomotive syndrome in the elderly: The 25-question Geriatric Locomotive Function Scale. Journal of Orthopaedic Science. 2012; 17(2):163-72. [DOI:10.1007/s00776-011-0193-5] [PMID]

[15] Sadeghi Mahali N, Hosseini M, Rahgozar M, Nourozi Tabrizi k. Evaluation of cultural adaptation, validity and reliability of the questionnaire of Geriatric Locomotive Function Scale - 25 questions. Pharmacophore. 2017; 8(6S):e1173929. https://pharmacophorejournal.com/m5ZYMwm

[16] Sharifi H, Sharifi N. [Principles of psychometrics and psychosis (Persian)]. Tehran: Roshd; 2015. http://opac.nlai.ir/ opac-prod/search/briefListSearch.do?_author

[17] Sayari A-A. Elders condition in Iran. Tehran: ISNA; 2015. https://www.isna.ir/news/94062918260/

[18] Noge S, Ohishi T, Yoshida T, Kumagai H. Quantitative assessment of locomotive syndrome by the loco-check questionnaire in older Japanese females. Journal of Physical Therapy Science. 2017; 29(9):1630-6. [DOI:10.1589/jpts.29.1630] [PMID] [PMCID]

[19] Ishibashi H. Locomotive syndrome in Japan. Osteoporosis and Sarcopenia. 2018; 4(3):86-94. [DOI:10.1016/j. afos.2018.09.004] [PMID] [PMCID]

[20] Fujita N, Sakurai A, Miyamoto A, Michikawa T, Tsuji O, Nagoshi $\mathrm{N}$, et al. Lumbar spinal canal stenosis leads to locomotive syndrome in elderly patients. Journal of Orthopaedic Science. 2019; 24(1):19-23. [DOI:10.1016/j.jos.2018.08.004] [PMID]

[21] Endo N. Osteoporosis as a cause of locomotive syndrome: The influence on functional mobility and activities of daily living. Clinical Reviews in Bone and Mineral Metabolism. 2016; 14(2):74-6. [DOI:10.1007/s12018-016-9215-3]

[22] Momoki C, Habu D, Ogura J, Tada A, Hasei A, Sakurai K, et al. Relationships between sarcopenia and household status and locomotive syndrome in a community-dwelling elderly women in Japan. Geriatrics \& Gerontology International. 2017; 17(1):54-60. [DOI:10.1111/ggi.12674] [PMID]

[23] Kim Y, Suehara Y, Ishii M, Kawasaki T, Matsuoka K, Okubo T, et al. A comparative study of 2 screening tools for locomotive syndrome (The "Loco-check" and the "GLFS$\left.25^{\prime \prime}\right)$ : An orthopedic outpatient-based survey. Journal of $\mathrm{Ad}$ vances in Medicine and Medical Research. 2016; 17(5):1-13. [DOI:10.9734/BJMMR/2016/28194] 